\title{
Effects of caloric restriction on the expression of lipocalin-2 and its receptor in the brown adipose tissue of high-fat diet-fed mice
}

\author{
Kyung-Ah Park' , Zhen Jin', Hyeong Seok An ${ }^{1}$, Jong Youl Lee', Eun Ae Jeong ${ }^{1}$, Eun Bee Choi ${ }^{1}$, Kyung Eun Kim', \\ Hyun Joo Shin', Jung Eun Lee', and Gu Seob Roh ${ }^{1 * *}$ \\ ${ }^{1}$ Department of Anatomy and Convergence Medical Science, Bio Anti-Aging Medical Research Center, ${ }^{2}$ Department of Thoracic and Cardiovascular Surgery, \\ Institute of Health Sciences, College of Medicine, Gyeongsang National University, Jinju 52727, Korea
}

\section{ARTICLE INFO}

Received May 2, 2019

Revised June 25, 2019

Accepted July 9, 2019

*Correspondence

Gu Seob Roh

E-mail: anaroh@gnu.ac.kr

Key Words

Brown adipose tissue

Caloric restriction

Lipocalin 2

Obesity

\begin{abstract}
Obesity causes inflammation and impairs thermogenic functions in brown adipose tissue (BAT). The adipokine lipocalin 2 (LCN2) has been implicated in inflammation and obesity. Herein, we investigated the protective effects of caloric restriction (CR) on LCN2-mediated inflammation and oxidative stress in the BAT of high-fat diet (HFD)-fed mice. Mice were fed a HFD for 20 weeks and then either continued on the HFD or subjected to CR for the next 12 weeks. CR led to the browning of the white fat-like phenotype in HFD-fed mice. Increased expressions of LCN2 and its receptor in the BAT of HFD-fed mice were significantly attenuated by CR. Additionally, HFD+CR-fed mice had fewer neutrophils and macrophages expressing LCN2 and iron-positive cells than HFD-fed mice. Further, oxidative stress and mitochondrial fission induced by a HFD were also significantly attenuated by CR. Our findings indicate that the protective effects of CR on inflammation and oxidative stress in the BAT of obese mice may be associated with regulation of LCN2.
\end{abstract}

\section{INTRODUCTION}

Brown adipose tissue (BAT) is characterized by multilocular lipid droplets, mitochondria-rich adipocytes, and the expression of uncoupling protein 1 (UCP1) [1,2]. BAT is more resistant to obesity-induced inflammation and oxidative stress than white adipose tissue (WAT) [3]. However, reductions in BAT function dramatically contribute to the development of obesity and/or diabetes $[4,5]$, and BAT dysfunction has been associated with inflammation and oxidative stress in both of these conditions $[6,7]$. Therefore, BAT may be a useful target for treatments of obesity and/or diabetes [8]. However, more precise mechanisms are required for the amelioration of obesity-induced BAT dysfunction.

Lipocalin 2 (LCN2) is a critical regulator of BAT activation $[9,10]$. Its deficiency leads to an increase in body weight and impairs adaptive thermogenesis [11]. In response to cold, LCN2 upregulates the expression of UCP1, which mediates BAT ther- mogenic activity $[9,10]$. Notably LCN2, which was originally isolated from neutrophilic granules, has been linked to obesity and adipocyte inflammation because nuclear factor-kappa B (NF- $\mathrm{B})$ transactivates its expression $[12,13]$. Furthermore, LCN2 is believed to be an iron-binding protein [14] that can induce oxidative stress [15]. Based on these findings, we propose that LCN2 plays an important role in the regulation of iron-mediated inflammation and oxidative stress in the BAT of obese mice.

Caloric restriction (CR) has been shown to reverse obesityinduced insulin resistance by reducing adiposity [16] and increasing mitochondrial biogenesis [17]. However, its effects on inflammation and oxidative stress in mice fed a high-fat diet (HFD) are unclear. Hence, we investigated the protective effects of CR on LCN2-mediated inflammation and oxidative stress in the BAT of HFD-fed mice.
This is an Open Access article distributed under the terms of the Creative Commons Attribution Non-Commercial License, which permits unrestricted non-commercial use, distribution, and reproduction in any medium, provided the original work is properly cited. Copyright @ Korean J Physiol Pharmacol, pISSN 1226-4512, elSSN 2093-3827
Author contributions: G.S.R. initiated the project and supervised the study. K.A.P., Z.J., H.S.A., J.Y.L., E.A.J., and E.B.C. performed experiments and data analyses. K.E.K., H.J.S., and J.E.L. discussed the interpretation of the data. K.A.P. and G.S.R. wrote the manuscript. 


\section{METHODS}

\section{Animal model}

Three-week-old male C57BL/6 mice were purchased from KOATECH (Pyeongtaek, Korea) and maintained in the animal facility at Gyeongsang National University (GNU). Animal experiments were performed in accordance with the National Institutes of Health guidelines on the use of laboratory animals. The GNU Animal Care Committee for Animal Research approved the study protocol (GNU-130306-M0021). The mice were individually housed under a 12-h light/dark cycle. The mice had ad libitum access to food and water. To determine the effects of CR on changes in BAT induced by a HFD, mice were divided into two groups at 4 weeks of age and fed either a HFD ( $\mathrm{n}=20,60 \mathrm{kcal} \%$ fat, $5.24 \mathrm{kcal} / \mathrm{g}$; Research Diet, Inc., New Brunswick, NJ, USA) or normal standard diet chow ( $\mathrm{n}=10$, normal diet [ND], $3.1 \mathrm{kcal} / \mathrm{kg}$; Harlan Laboratories, Inc., Indianapolis, IN, USA) for 20 weeks. HFD-fed animals were then either continued on the HFD ( $\mathrm{n}=$ 10 ) or subjected to CR ( $\mathrm{n}=10,2 \mathrm{~g}$ /day of the HFD) for 12 weeks, as previously described [18].

\section{Histological analysis and iron (III) staining}

For histological analyses and staining, mice ( $\mathrm{n}=4$ per group) were anesthetized with tiletamine/zolazepam (Zoletil $5 \mathrm{mg} / \mathrm{kg}$; Virbac Laboratories, Carros, France). BAT samples from the interscapular region were fixed with $4 \%$ paraformaldehyde in 0.1 $\mathrm{M}$ phosphate buffered saline. After $6 \mathrm{~h}$, BAT was embedded in paraffin, sliced into $5-\mu \mathrm{m}$ sections, and stained with hematoxylin and eosin (H\&E) (Sigma-Aldrich, St. Louis, MO, USA). The sections were visualized under a BX51 light microscope (Olympus, Tokyo, Japan). Iron accumulation in BAT was identified using an iron (III) staining kit (Abcam, Cambridge, UK), according to the manufacturer's instructions. Deposits of iron stained blue, and nuclei stained pink. Iron-positive cells were counted under light microscopy. Three fields $\left(500 \times 500 \mu \mathrm{m}^{2}\right)$ were randomly selected from two consecutive sections ( $n=4$ per group).

\section{BAT triglyceride assay}

Frozen BAT samples ( $\mathrm{n}=6$ per group) were homogenized, and triglyceride (TG) levels were determined using a colorimetric assay kit (Cayman, Ann Arbor, MI, USA), according to the manufacturer's protocol.

\section{Quantitative real-time polymerase chain reaction (qRT-PCR)}

Total BAT RNA ( $\mathrm{n}=6$ per group) was isolated using the TRIzol reagent (Invitrogen, Carlsbad, CA, USA). Complementary DNA was then synthesized using a reverse-transcription kit (Thermo
Scientific, Waltham, MA, USA), according to the manufacturer's instructions. QRT-PCR was performed using the LightCycler 480 Instrument II (Roche, Mannheim, Germany) with the TOPreal qPCR $2 \times$ PreMix (Enzynomics, Daejeon, Korea) and iQ SYBR Green Supermix (Bio-Rad, Hercules, CA, USA). The PCR primers used for this study were presented in Supplementary Table 1. Expression was normalized to the level of glyceraldehyde 3-phosphate dehydrogenase as an internal control.

\section{Protein extraction and Western blotting}

Total lysates were prepared from BAT ( $n=6$ per group), as previously described [19]. To obtain the nuclear fraction, we used the NE-PER Nuclear and Cytoplasmic Extraction Kit (Pierce, Rockford, IL, USA). Mitochondria from BAT was obtained using the Mitochondrial Isolation Kit (Thermo Fisher Scientific). Protein concentrations were determined using a Bio-Rad protein assay, and samples were stored at $-80^{\circ} \mathrm{C}$ until use. Western blot analyses were performed using standard methods [19]. Specific proteins were identified using primary antibodies (Supplementary Table 2). Alpha-tubulin was used as the reference protein for the total fraction, and p84 was used as a loading control for the nuclear fraction. Protein bands were detected by enhanced chemiluminescence (Pierce) and quantified using the Multi Gauge image analysis program (version 3.0; Fujifilm, Tokyo, Japan).

\section{Immunofluorescence}

For double immunofluorescence, deparaffinized sections were incubated with primary antibodies (Supplementary Table 2) overnight at $4^{\circ} \mathrm{C}$. After washing, the sections were incubated with Alexa Fluor 488- or Alexa 594-conjugated donkey anti-rabbit or anti-mouse secondary antibodies (Invitrogen Life Technologies, Carlsbad, CA, USA). Nuclei were counterstained with DAPI (Invitrogen). Fluorescent images were captured using a BX51-DSU microscope.

\section{Statistical analysis}

Prism software (GraphPad Software Inc., San Diego, CA, USA) was used for the statistical analysis. One-way analysis of variance followed by Bonferroni's post hoc test was used for group comparisons. A p-value of less than 0.05 was indicative of statistical significance.

\section{RESULTS}

\section{Effects of CR on HFD-induced white adipocyte-like phenotype in BAT}

To examine the effect of CR on HFD-induced white adipocyte- 
like phenotype, we used our established mouse model of CR (Fig. 1A) [18]. Body weight was significantly lower in HFD+CRtreated mice $(36.1 \pm 0.50)$ than in HFD-fed mice $(54.8 \pm 0.93)$ but was similar to levels observed in ND-fed mice (40.4 \pm 0.70$)$. H\&E staining showed that CR decreased the number of large unilocular adipocytes in the BAT of HFD-fed mice (Fig. 1B). Using immunofluorescence staining, we observed that the number of large perilipin-positive adipocytes in HFD-fed mice was reduced by CR (Fig. 1B). Western blot analysis also showed that CR increased the reduced expression of perilipin in the BAT of HFD-treated mice (Fig. 1C). In addition, the increase in TG storage in the BAT of HFD-fed mice was attenuated by CR (Fig. 1D).

\section{Effects of CR on LCN2 expression and iron accumulation in the BAT of HFD-fed mice}

Because LCN2 is upregulated in fat cells in obese rodents and regulates BAT activation [9,10], we examined the effects of CR on the expression of LCN2 and its receptor (24p3R) in the BAT of HFD-fed mice. HFD induction of LCN2 and 24p3R expression was significantly attenuated by CR (Fig. 2A, B). Consistent with this finding, we found less immunostaining of LCN2-positive neutrophil elastase in the BAT of HFD+CR-fed mice compared with HFD-fed mice (Fig. 2C). Furthermore, because LCN2 is known to facilitate the transport of siderophore-iron complexes into cells [14] and induce oxidative stress [15], we assessed BAT iron accumulation using an iron staining kit. CR reduced the number of iron-positive cells in the BAT of HFD-fed mice (Fig. 2D, E).
A

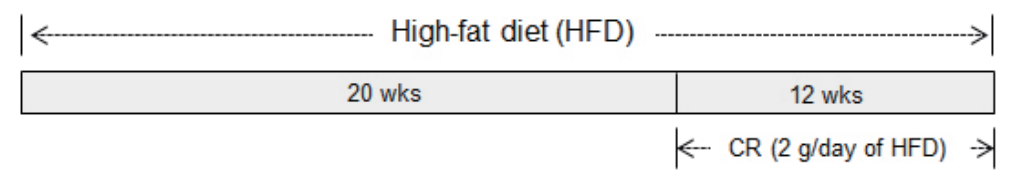

B

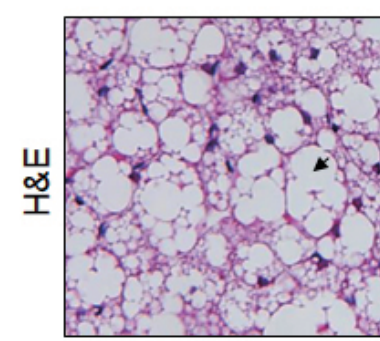

ND
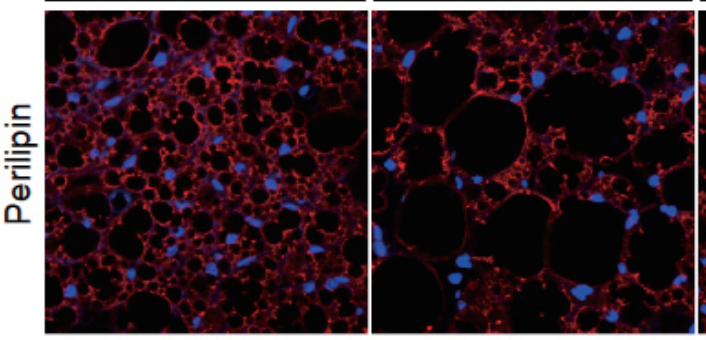

C
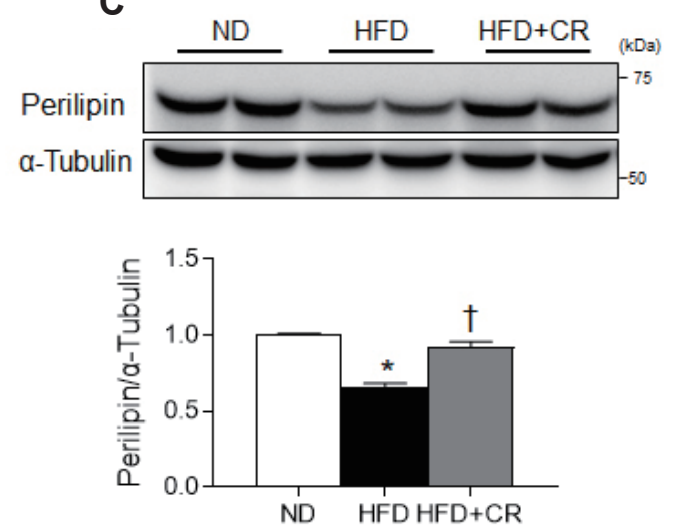

HFD
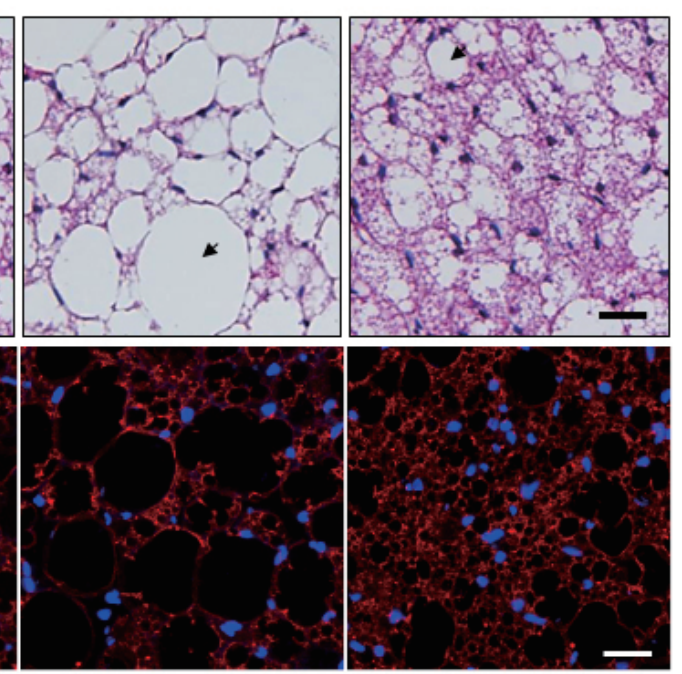

D

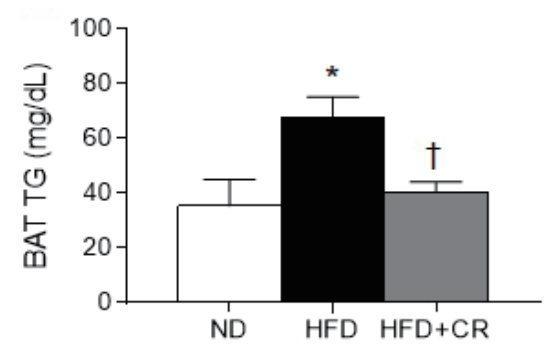

Fig. 1. Effects of caloric restriction (CR) on high-fat diet (HFD)-induced white adipocyte-like phenotype in brown adipose tissue (BAT). (A) Schematic representation of experimental protocol. Male C57/6 mice were fed HFD for 20 weeks and then continued on the HFD or subjected to CR ( $2 \mathrm{~g} /$ day of HFD) for 12 weeks. (B) Representative images ( $\times 200)$ of H\&E staining (upper panel) and immunofluorescence for perilipin (lower panel) in BAT sections. Nuclei were counterstained with DAPI. Arrow indicates lipid droplet. (C) Western blot analysis of perilipin protein expression using $\alpha$-tubulin as a reference protein. (D) Triglyceride concentration in BAT. Data are presented as mean \pm standard error of the mean; ${ }^{*} p<0.05$ vs. normal $\operatorname{diet}(\mathrm{ND})$-fed mice; ${ }^{\dagger} \mathrm{p}<0.05 \mathrm{vs}$. HFD-fed mice. Scale bar $=25 \mu \mathrm{m}$. 

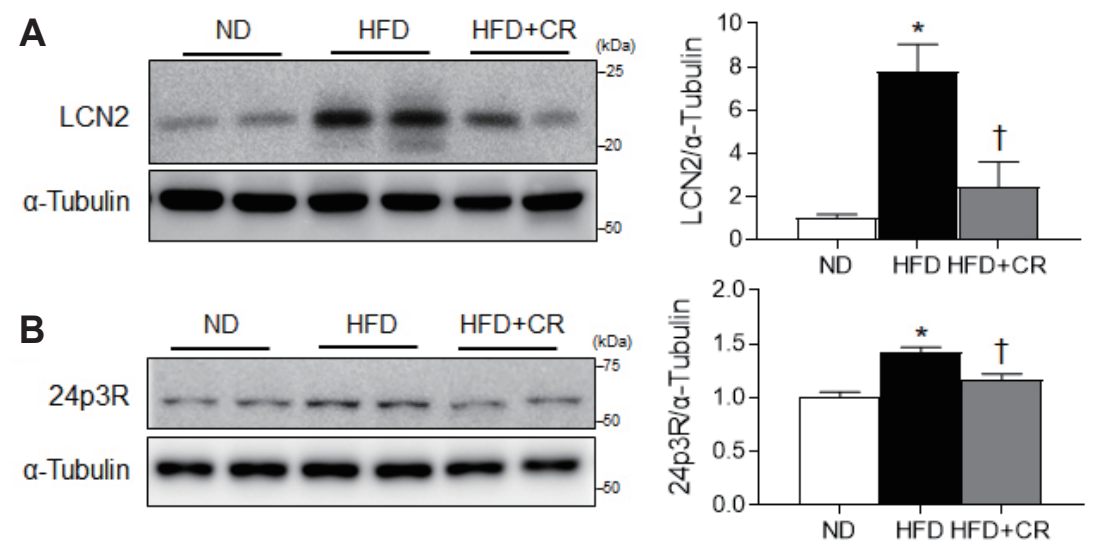

C

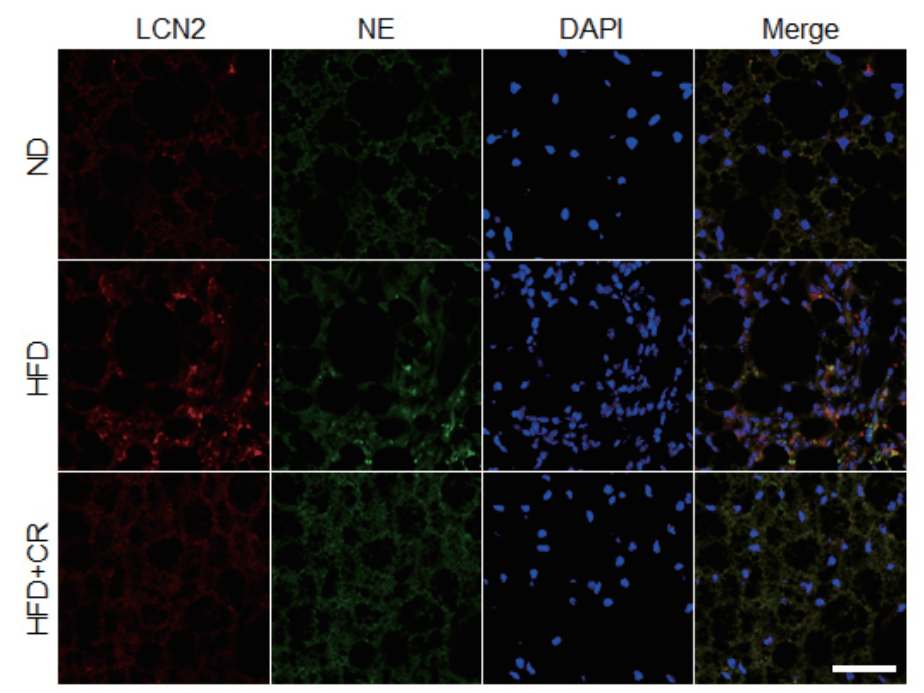

D

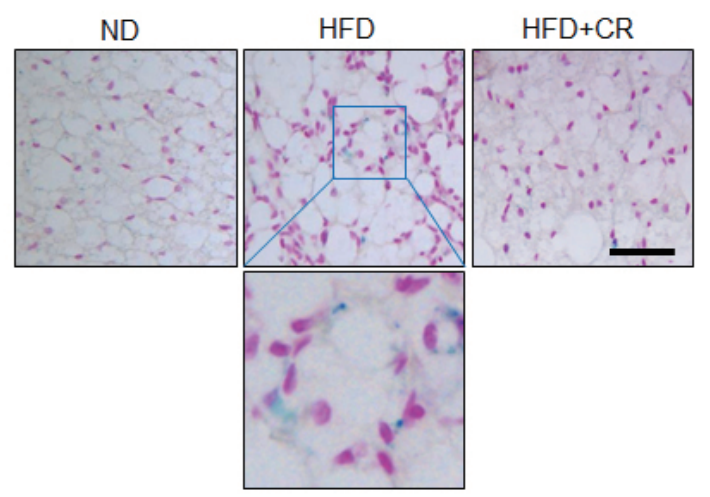

E

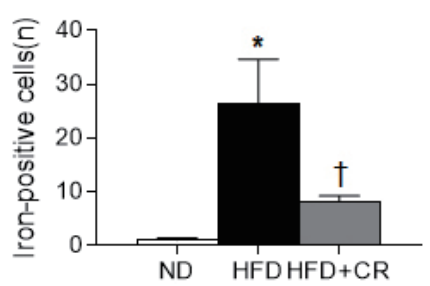

Fig. 2. Effects of caloric restriction (CR) on lipocalin 2 (LCN2), 24p3R expression, and iron accumulation in the brown adipose tissue (BAT) of highfat diet (HFD)-fed mice. (A, B) Western blot analysis of LCN2 and 24p3R protein expression using $\alpha$-tubulin as a reference protein. (C) Representative images $(\times 200)$ of immunofluorescence for LCN2 and neutrophil elastase (NE) in BAT sections. Nuclei were counterstained with DAPI. (D) Representative images ( $\times 200)$ of iron staining in BAT sections. (E) The number of iron-positive cells in BAT sections. Data are presented as mean \pm standard error of the mean; ${ }^{*} p<0.05 v s$. normal diet (ND)-fed mice; ${ }^{\dagger} p<0.05 v s$. HFD-fed mice. Scale bar $=50 \mu \mathrm{m}$.

\section{Effects of CR on inflammation and oxidative stress in the BAT of HFD-fed mice}

LCN2 is a known regulator of macrophage polarization and $N F-\kappa B$ pathway activation [20]. We observed that the number of infiltrating F4/80-positive macrophages and LCN2-positive neutrophils were higher in the BAT from HFD-fed mice than from ND-fed mice (Fig. 3A). However, there were significantly fewer co-localized LCN2-positive macrophages and neutrophils in HFD+CR-treated mice compared with HFD-fed mice (Fig. 3A).
In addition, nuclear NF- $\mathrm{Bp} 65$ expression in HFD-fed mice was significantly reduced by CR (Fig. 3B).

Oxidative stress is an important feature of HFD-induced obesity [21]. Nuclear factor erythroid 2-related factor 2 (Nrf2) and heme oxygenase-1 (HO-1) protect against oxidative injury [22], whereas mitochondrial ferritin (FtMt), an iron-storage protein in mitochondria, protects these organelles from iron-induced oxidative stress by sequestering excess free iron [23]. Here, we found that the protein expression levels of Nrf2, HO-1, and superoxide dismutase1 (SOD1) were significantly higher in HFD-fed than 
A

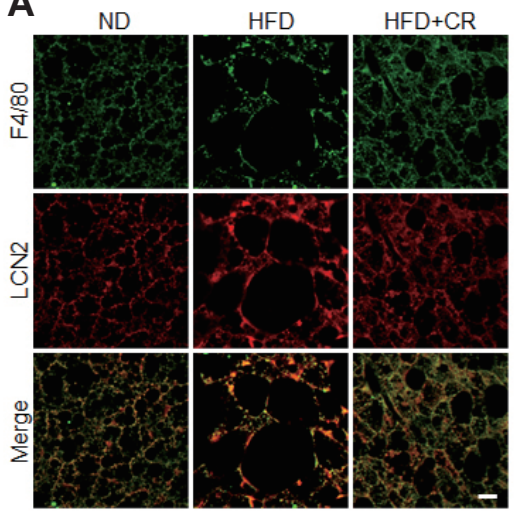

C

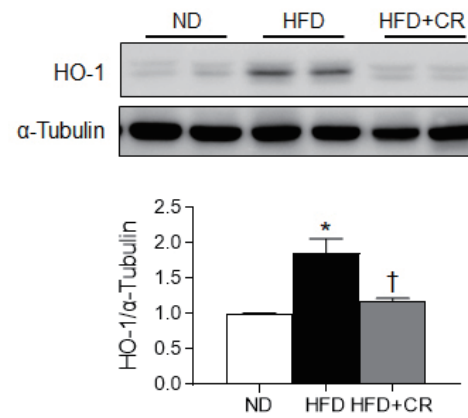

E

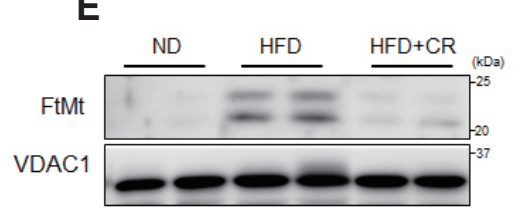

B

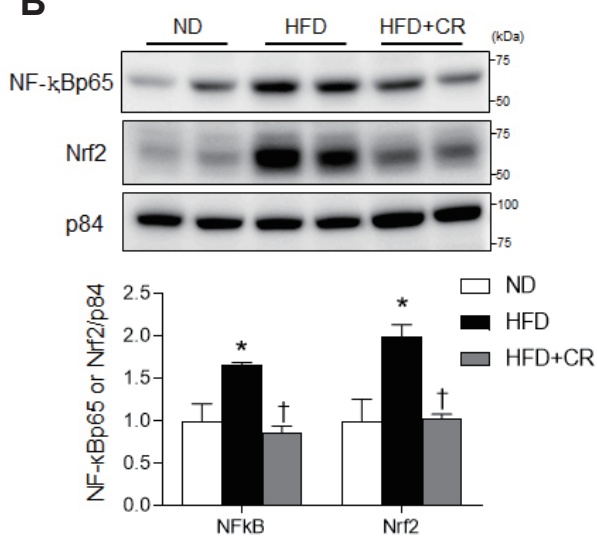

D
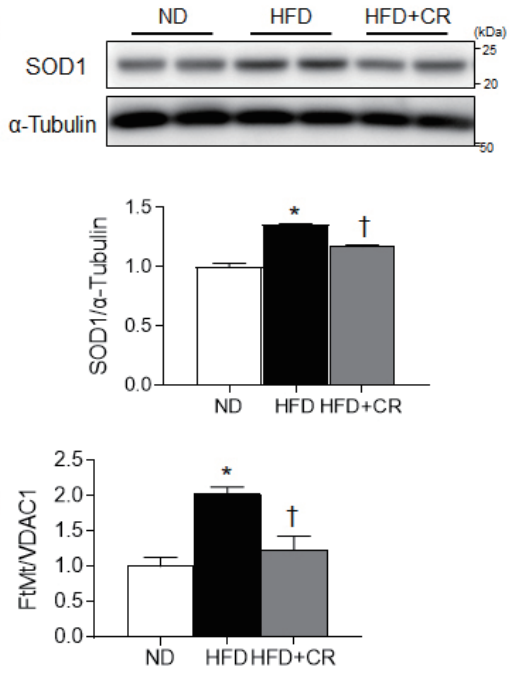

Fig. 3. Effects of caloric restriction (CR) on inflammation and oxidative stress in the brown adipose tissue (BAT) of high-fat diet (HFD)-fed mice. (A) Representative images $(\times 200)$ of immunofluorescence for $\mathrm{F} 4 / 80$ and lipocalin 2 (LCN2) in BAT sections. (B) Western blot analysis of nuclear factor-kappa B (NF$\kappa \mathrm{B})$ and nuclear respiratory factor-2 (Nrf2) protein expression, using p84 as a reference protein. (C, D) Western blot analysis of heme oxygenase-1 (HO-1) and superoxide dismutase1 (SOD1) protein, using $\alpha$-tubulin as a reference protein. (E) Western blot analysis of mitochondrial ferritin (FtMt) protein expression, using voltagedependent anion selective channel 1 (VDAC1) as a reference protein. Data are presented as mean \pm standard error of the mean; ${ }^{*} p<0.05$ vs. normal diet (ND)fed mice; ${ }^{\dagger} p<0.05$ vs. HFD-fed mice. Scale bar $=10 \mu \mathrm{m}$.
ND-fed mice, and that these upregulated levels were significantly attenuated by CR (Fig. 3B-D). Additionally, the expression of FtMt was significantly higher in HFD-fed mice but was normalized by CR (Fig. 3E).

\section{Effects of CR on mitochondrial fission in the BAT of HFD-fed mice}

The accumulation of excess lipids in adipocytes is associated with mitochondrial fission [24], and hyperglycemia-induced ROS is associated with dramatic changes in mitochondrial morphology $[25,26]$. CR significantly attenuated the increased expression of dynamin-related protein1 (Drp1) and phosphorylated Drp1 (pDrpl) in HFD-fed mice (Fig. 4A). In addition, decreased cytosolic Drpl expression in the BAT of HFD-fed mice is reversed by CR (Fig. 4B). Immunofluorescence results showed that CR reduced the number of pDrpl-positive cells in the BAT of HFD-fed mice (Fig. 4C).

\section{Effects of CR on UCP1 expression in the BAT of HFD- fed mice}

Because UCP1 activity in adipocytes is associated with mitochondrial fission [24], we investigated the effects of CR on UCP1 expression in the BAT of HFD-fed mice. Unexpectedly, HFD-fed mice subjected to CR had significantly higher UCP1 protein levels than HFD-fed mice (Fig. 5A). In addition, immunofluorescence results showed that a high level of UCP1 immunoreactivity was present in the BAT of HFD+CR-treated mice compared with HFD-fed mice (Fig. 5B). Furthermore, to demonstrate the effects of $\mathrm{CR}$ on the browning of the white adipocyte-like phenotype in the BAT of HFD-treated mice, we determined the mRNA expression levels of browning marker genes, such as Ucpl, peroxisome proliferator-activated receptor gamma coactivator 1 alpha $(P g c l \alpha)$, cell-death-inducing DFFA-like effector A (Cidea), and PR domain-containing 16 (Prdm16) (Fig. 5C). Of interest, a HFD had no effect on the expression of other BAT marker genes except for Ucpl. 

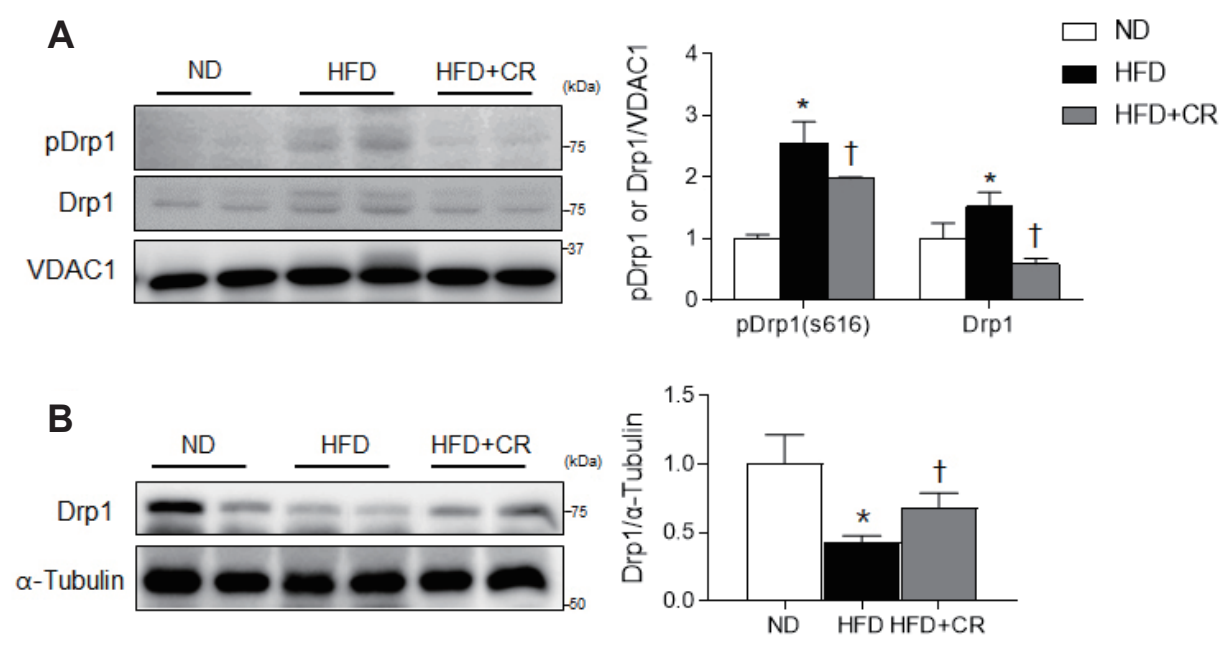

C

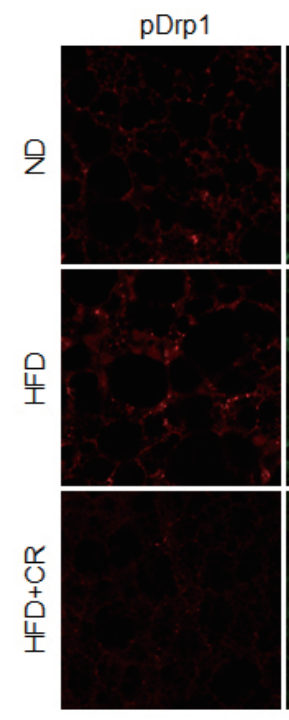

VDAC1
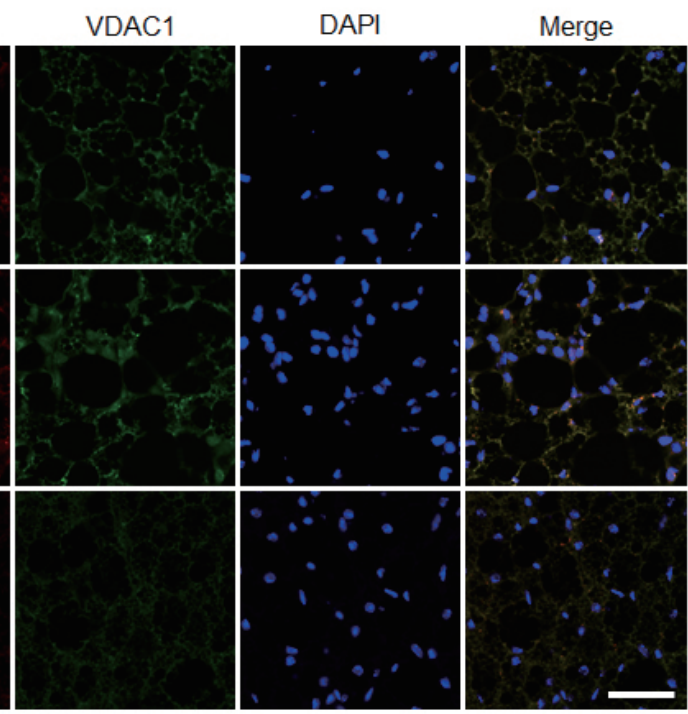

Fig. 4. Effects of caloric restriction (CR) on mitochondrial fission in the brown adipose tissue (BAT) of high-fat diet (HFD)-fed mice. (A) Western blot analysis of phosphorylated dynamin like protein1 (pDrp1) (s616) and dynamin like protein1 (Drp1) protein expression, using voltage-dependent anion selective channel 1 (VDAC1) as a reference. (B) Western blot analysis of cytosolic Drp1 protein levels using $\alpha$-tubulin as a reference protein. (C) Representative images $(\times 200)$ of immunofluorescence for pDrp1 and VDAC1 in BAT sections. Nuclei were counterstained with DAPI. Data are presented as mean \pm standard error of the mean; ${ }^{*} p<0.05$ vs. normal diet (ND)-fed mice; ${ }^{\dagger} p<0.05 v$ s. HFD-fed mice. Scale bar $=50 \mu \mathrm{m}$.

\section{DISCUSSION}

Numerous studies have established that CR greatly improves insulin sensitivity and thermogenic capacity in obese subjects $[27,28]$. In the present study, mice fed a HFD caused the BAT to exhibit a white adipocyte-like phenotype and manifest higher levels of macrophage infiltration, NF- $\mathrm{KB}$ expression, antioxidant enzyme activity, and pDrpl expression. CR was shown to counter these effects, lowering HFD-induced inflammation, oxidative stress, and mitochondrial fission, but increasing UCP1 levels. Furthermore, CR attenuated HFD-induced LCN2 and 24p3R expression in the BAT of HFD-fed obese mice. Thus, our findings suggest CR prevents BAT dysfunction through multiple mechanisms by reducing inflammation, oxidative stress, and mitochondrial fission in BAT.

Obesity is characterized by ectopic fat accumulation, macrophage infiltration, apoptosis, oxidative stress, and mitochondrial dysfunction in WAT $[29,30]$. A HFD dramatically increases TG storage in lipid droplets in both WAT and BAT. In WAT, catecholamine-stimulated lipolysis liberates fatty acids for use as fuel during HFD feeding. In contrast, lipolysis in BAT provides fatty acids for heat production by adaptive thermogenesis in response to overfeeding or a cold environment [31,32]. Perilipin is the predominant lipid droplet coat protein in fat cells and plays an important role in the regulation of lipolysis and thermogenesis [33]. Perilipin-null mice are more susceptible to insulin resistance following HFD feeding [34]; it has been reported that the overexpression of perilipin causes a reduction in lipid droplet size in WAT, resembling a more BAT-like morphology [35]. Consistent with these observations, our present study showed that an increase in adiposity within the BAT of HFD-treated mice is associated with a significant reduction in perilipin expression and an increase in the size of lipid droplets [36]. However, this reduction in perilipin expression in the BAT of HFD-fed mice was largely reversed by CR. Thus, these findings suggest that perilipin may be important for lipid storage, lipolysis, and adaptive thermogenesis in BAT.

Circulating LCN2 levels positively correlate with fat mass and 


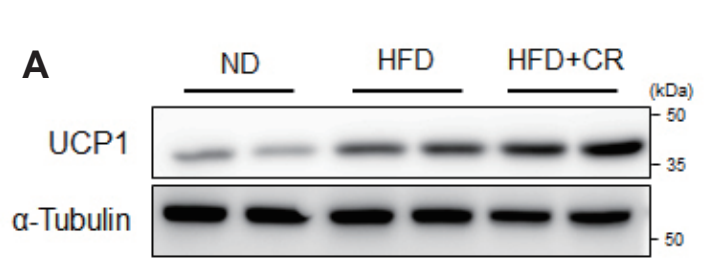

B
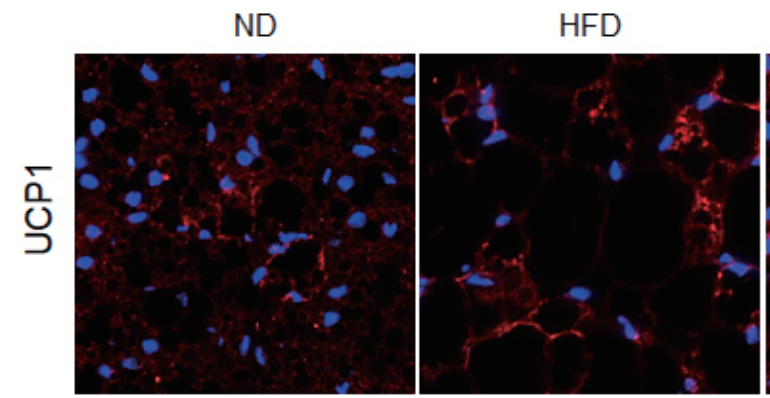

C

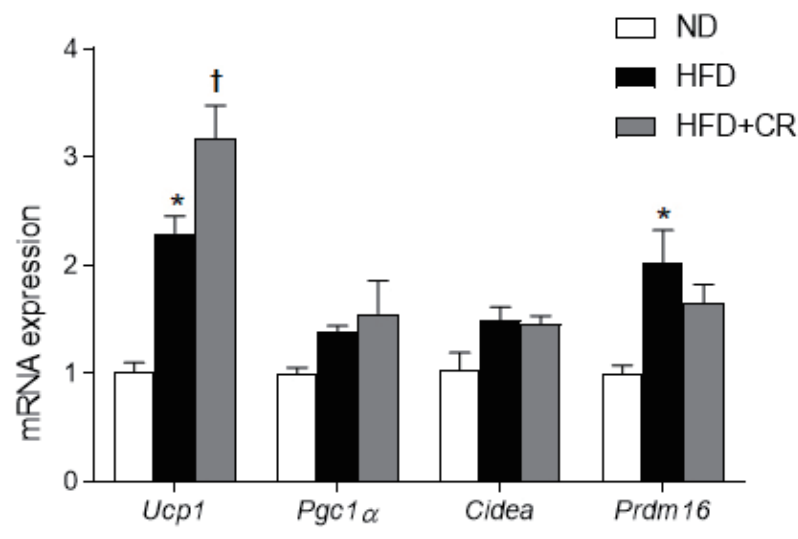

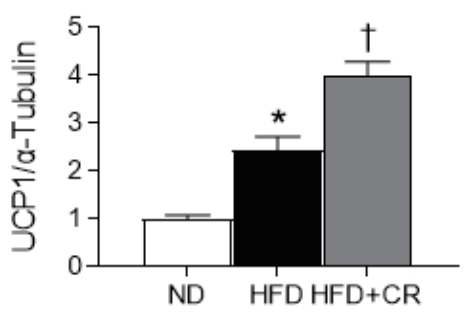

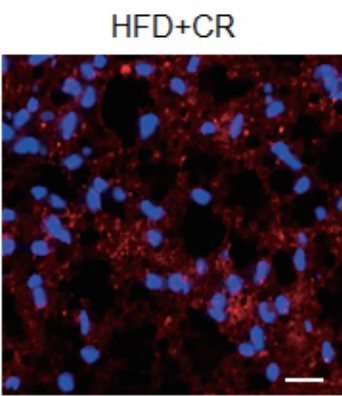

Fig. 5. Effects of caloric restriction (CR) on uncoupling protein 1 (UCP1) expression in the brown adipose tissue (BAT) of high-fat diet (HFD)-fed mice. (A) Western blot analysis of UCP1 protein expression, using $\alpha$-tubulin as a reference protein. (B) Representative images $(\times 200)$ of immunofluorescence for UCP1 in BAT sections. Nuclei were counterstained with DAPI. (C) mRNA expression of BAT-selective genes from BAT samples using qRT-PCR. Data are presented as mean \pm standard error of the mean; ${ }^{*} p<$ 0.05 vs. normal diet (ND)-fed mice; ${ }^{\dagger} p<$ $0.05 v s$. HFD-fed mice. Scale bar $=10 \mu \mathrm{m}$. insulin resistance in obese humans and genetically obese mice [37]. Our previous study demonstrated that CR attenuated the increase in serum LCN2 levels in db/db mice [38]. In the present study, we found that CR reversed the HFD-induced increase in LCN2 and 24p3R expression in BAT. These data indicate that the CR-induced reduction in the fat mass of HFD-fed mice may be responsible for the low secretion of LCN2 from adipose tissues. However, other adrenergic-independent mechanisms may be involved in the observed changes in thermogenic function and oxidative stress.

LCN2 induction is activated by the proinflammatory cytokine interleukin-1 $\beta$, which is induced by the NF-кB pathway [39]. In obesity, the excessive levels of lipids released by adipocytes have been known to promote the recruitment of M1-polarized macrophages in WAT [40]. In this study, we showed that CR significantly reduces the HFD induction of nuclear NF- $\mathrm{Bp} 65$ expression and F4/80-positive macrophage infiltration in BAT. These findings are consistent with evidences suggesting that LCN2 promotes inflammation in lipopolysaccharide (LPS)treated microglia in the brain or tumor necrosis factor-a-induced 3T3-L1 adipocytes or in mice fed with a HFD for 18 weeks [41-
43]. However, several subsequent studies of Guo et al. reported that LCN2-deficient mice exhibit increased levels of M1 markers in LPS-treated adipose tissue as anti-inflammatory regulators of macrophage activation [9-11,20]. Thus, these disputes have been ongoing for the role of LCN2 in obesity or inflammatory conditions. We suggest that there are two possible factors to consider that could explain such widely discordant phenotypes. First, there are differences in generating LCN2 deficient mice. Guo et al. [10] used LCN2 knockout (KO) mice, in which exon 2 through 5 were deleted, whereas Law et al. [43] used LCN2 KO mice, in which exon 1 through 6 were deleted. Second, these discrepancies could result from the differences in the doses of LPS or duration of HFD used in animal studies or in the types of cells and tissues examined.

LCN2, an iron transport protein, has been shown to be involved in iron overload-mediated oxidative stress and NF- $\kappa \mathrm{B}-$ dependent inflammation in various experimental models of obesity $[20,39]$. Cardiomyocytes, pancreatic $\beta$ cells, and BAT are all metabolically active tissues that can develop iron-based cellular pathologies [44,45]. Specifically, obesity and diabetes are known to be associated with iron overload [45]. Here, we showed that CR 
is associated with reduction in LCN2 protein expression and iron accumulation in the BAT of HFD-fed obese mice. Iron overload causes oxidative stress and the induction of adaptive antioxidant responses involving Nrf2, HO-1, and SOD1 in the BAT of HFDfed mice. Consistent with the results of previous studies demonstrating that $\mathrm{CR}$ can ameliorate obesity-induced oxidative stress $[46,47]$, we showed that CR reduces the expression levels of Nrf2 and FtMt, which protect against iron-induced oxidative stress [47]. Iron sequestration by FtMt is likely to be important in countering the toxicity caused by the accumulation of iron associated with chronic HFD feeding. Thus, the increases in iron overload and FtMt expression in BAT may be linked to the protective role of FtMt against iron-induced oxidative stress. Based on these findings, we hypothesize that iron overload-induced oxidative stress may be a key component of the inflammation and mitochondrial dysfunction linked to obesity and diabetes.

In general, obesity is associated with increases in mitochondrial fission, leading to fragmentation, which may be an adaptive cellular response [26]. Pisani et al. [24] recently reported that mitochondrial fission is associated with UCP1 activity in beige adipocytes. The present work is consistent with HFD-induced mitochondrial fission in BAT being associated with physiological adaptive thermogenesis rather than being a deleterious outcome. During HFD feeding, increases in mitochondrial fission provide a greater capacity for oxidation and thermogenesis in BAT, whose activation requires high expression levels of UCP1. These findings are also consistent with previous findings that HFD-induced adaptive thermogenesis in BAT involves the upregulation of UCP1 [3]. Although CR significantly attenuated HFD-induced Drp1 phosphorylation in BAT, HFD-induced upregulation of UCP1 expression was further increased by CR. This finding suggests that this overall decrease in total fat during CR is due, at least in part, to the increased browning of white fat depots in HFD-fed mice [27]. Despite CR resulting in higher UCP1 mRNA and protein levels, the transcriptional levels of other brown fat-specific genes (i.e., Pgcl $\alpha$, Cidea, and Prdm16) were all unchanged in the BAT of HFD-fed mice, suggesting there were defects in BAT functions. Maybe, these findings indicate that $\mathrm{CR}$ could not completely reverse BAT function for metabolic thermogenesis from white adipocyte-like BAT to browning BAT.

In conclusion, this study demonstrated that BAT activation responds to a HFD by regulating metabolic thermogenesis. Furthermore, $\mathrm{CR}$ attenuates NF- $\kappa \mathrm{B}$-dependent inflammation, iron overload-mediated oxidative stress, and mitochondrial fission in the BAT of HFD-fed mice. These findings suggest that CR can prevent obesity-associated metabolic disturbances by regulating LCN2-associated inflammation and oxidative stress in BAT.

\section{ACKNOWLEDGEMENTS}

We thank Chin-ok Yi for technical support. This study was sup- ported by a National Research Foundation of Korea grant, funded by the Korean government (grant no. 2015R1A5A2008833).

\section{CONFLICTS OF INTEREST}

The authors declare no conflicts of interest.

\section{SUPPLEMENTARY MATERIALS}

Supplementary data including two tables can be found with this article online at http://pdf.medrang.co.kr/paper/pdf/Kjpp/ Kjpp2019-23-05-07-s001.pdf.

\section{REFERENCES}

1. Farmer SR. Molecular determinants of brown adipocyte formation and function. Genes Dev. 2008;22:1269-1275.

2. Parker N, Crichton PG, Vidal-Puig AJ, Brand MD. Uncoupling protein-1 (UCP1) contributes to the basal proton conductance of brown adipose tissue mitochondria. J Bioenerg Biomembr. 2009;41:335342.

3. Alcalá M, Calderon-Dominguez M, Bustos E, Ramos P, Casals N, Serra D, Viana M, Herrero L. Increased inflammation, oxidative stress and mitochondrial respiration in brown adipose tissue from obese mice. Sci Rep. 2017;7:16082.

4. Lowell BB, S-Susulic V, Hamann A, Lawitts JA, Himms-Hagen J, Boyer BB, Kozak LP, Flier JS. Development of obesity in transgenic mice after genetic ablation of brown adipose tissue. Nature. 1993;366:740-742.

5. Franssens BT, Hoogduin H, Leiner T, van der Graaf Y, Visseren FLJ. Relation between brown adipose tissue and measures of obesity and metabolic dysfunction in patients with cardiovascular disease. $J$ Magn Reson Imaging. 2017;46:497-504.

6. Poher AL, Altirriba J, Veyrat-Durebex C, Rohner-Jeanrenaud F. Brown adipose tissue activity as a target for the treatment of obesity/ insulin resistance. Front Physiol. 2015;6:4.

7. Kim SH, Plutzky J. Brown fat and browning for the treatment of obesity and related metabolic disorders. Diabetes Metab J. 2016;40:12-21.

8. McGregor RA, Kwon EY, Shin SK, Jung UJ, Kim E, Park JH, Yu R, Yun JW, Choi MS. Time-course microarrays reveal modulation of developmental, lipid metabolism and immune gene networks in intrascapular brown adipose tissue during the development of dietinduced obesity. Int J Obes (Lond). 2013;37:1524-1531.

9. Zhang Y, Guo H, Deis JA, Mashek MG, Zhao M, Ariyakumar D, Armien AG, Bernlohr DA, Mashek DG, Chen X. Lipocalin 2 regulates brown fat activation via a nonadrenergic activation mechanism. J Biol Chem. 2014;289:22063-22077.

10. Guo H, Jin D, Zhang Y, Wright W, Bazuine M, Brockman DA, Bernlohr DA, Chen X. Lipocalin-2 deficiency impairs thermogenesis and potentiates diet-induced insulin resistance in mice. Diabetes. 2010;59:1376-1385.

11. Guo H, Foncea R, O'Byrne SM, Jiang H, Zhang Y, Deis JA, Blaner 
WS, Bernlohr DA, Chen X. Lipocalin 2, a regulator of retinoid homeostasis and retinoid-mediated thermogenic activation in adipose tissue. J Biol Chem. 2016;291:11216-11229.

12. Kjeldsen L, Johnsen AH, Sengeløv H, Borregaard N. Isolation and primary structure of NGAL, a novel protein associated with human neutrophil gelatinase. J Biol Chem. 1993;268:10425-10432.

13. Cowland JB, Muta T, Borregaard N. IL-1beta-specific up-regulation of neutrophil gelatinase-associated lipocalin is controlled by IkappaB-zeta. J Immunol. 2006;176:5559-5566.

14. Yang J, Goetz D, Li JY, Wang W, Mori K, Setlik D, Du T, ErdjumentBromage H, Tempst P, Strong R, Barasch J. An iron delivery pathway mediated by a lipocalin. Mol Cell. 2002;10:1045-1056.

15. Roudkenar MH, Kuwahara Y, Baba T, Roushandeh AM, Ebishima S, Abe S, Ohkubo Y, Fukumoto M. Oxidative stress induced lipocalin 2 gene expression: addressing its expression under the harmful conditions. J Radiat Res. 2007;48:39-44.

16. Dubé JJ, Amati F, Toledo FG, Stefanovic-Racic M, Rossi A, Coen $\mathrm{P}$, Goodpaster BH. Effects of weight loss and exercise on insulin resistance, and intramyocellular triacylglycerol, diacylglycerol and ceramide. Diabetologia. 2011;54:1147-1156.

17. Nisoli E, Tonello C, Cardile A, Cozzi V, Bracale R, Tedesco L, Falcone S, Valerio A, Cantoni O, Clementi E, Moncada S, Carruba MO. Calorie restriction promotes mitochondrial biogenesis by inducing the expression of eNOS. Science. 2005;310:314-317.

18. Kim H, Kang H, Heo RW, Jeon BT, Yi CO, Shin HJ, Kim J, Jeong SY, Kwak W, Kim WH, Kang SS, Roh GS. Caloric restriction improves diabetes-induced cognitive deficits by attenuating neurograninassociated calcium signaling in high-fat diet-fed mice. J Cereb Blood Flow Metab. 2016;36:1098-1110.

19. Jeong EA, Jeon BT, Shin HJ, Kim N, Lee DH, Kim HJ, Kang SS, Cho GJ, Choi WS, Roh GS. Ketogenic diet-induced peroxisome proliferator-activated receptor- $\gamma$ activation decreases neuroinflammation in the mouse hippocampus after kainic acid-induced seizures. Exp Neurol. 2011;232:195-202.

20. Guo H, Jin D, Chen X. Lipocalin 2 is a regulator of macrophage polarization and NF-кB/STAT3 pathway activation. Mol Endocrinol. 2014;28:1616-1628.

21. Manna P, Jain SK. Obesity, oxidative stress, adipose tissue dysfunction, and the associated health risks: causes and therapeutic strategies. Metab Syndr Relat Disord. 2015;13:423-444.

22. Loboda A, Damulewicz M, Pyza E, Jozkowicz A, Dulak J. Role of Nrf2/HO-1 system in development, oxidative stress response and diseases: an evolutionarily conserved mechanism. Cell Mol Life Sci. 2016;73:3221-3247.

23. Eaton JW, Qian M. Molecular bases of cellular iron toxicity. Free Radic Biol Med. 2002;32:833-840.

24. Pisani DF, Barquissau V, Chambard JC, Beuzelin D, Ghandour RA, Giroud M, Mairal A, Pagnotta S, Cinti S, Langin D, Amri EZ. Mitochondrial fission is associated with UCP1 activity in human brite/ beige adipocytes. Mol Metab. 2018;7:35-44.

25. Yu T, Robotham JL, Yoon Y. Increased production of reactive oxygen species in hyperglycemic conditions requires dynamic change of mitochondrial morphology. Proc Natl Acad Sci U S A. 2006; 103:2653-2658.

26. Wada J, Nakatsuka A. Mitochondrial dynamics and mitochondrial dysfunction in diabetes. Acta Med Okayama. 2016;70:151-158.

27. Fabbiano S, Suárez-Zamorano N, Rigo D, Veyrat-Durebex C, Steva- novic Dokic A, Colin DJ, Trajkovski M. Caloric restriction leads to browning of white adipose tissue through type 2 immune signaling. Cell Metab. 2016;24:434-446.

28. Johnson ML, Distelmaier K, Lanza IR, Irving BA, Robinson MM, Konopka AR, Shulman GI, Nair KS. Mechanism by which caloric restriction improves insulin sensitivity in sedentary obese adults. Diabetes. 2016;65:74-84.

29. Saltiel AR, Olefsky JM. Inflammatory mechanisms linking obesity and metabolic disease. JClin Invest. 2017;127:1-4.

30. Patti ME, Corvera $S$. The role of mitochondria in the pathogenesis of type 2 diabetes. Endocr Rev. 2010;31:364-395.

31. Greenberg AS, Egan JJ, Wek SA, Garty NB, Blanchette-Mackie EJ, Londos C. Perilipin, a major hormonally regulated adipocyte-specific phosphoprotein associated with the periphery of lipid storage droplets. J Biol Chem. 1991;266:11341-11346.

32. Souza SC, Christoffolete MA, Ribeiro MO, Miyoshi H, Strissel KJ, Stancheva ZS, Rogers NH, D'Eon TM, Perfield JW 2nd, Imachi H, Obin MS, Bianco AC, Greenberg AS. Perilipin regulates the thermogenic actions of norepinephrine in brown adipose tissue. J Lipid Res. 2007;48:1273-1279.

33. Girousse A, Langin D. Adipocyte lipases and lipid droplet-associated proteins: insight from transgenic mouse models. Int J Obes (Lond). 2012;36:581-594.

34. Saha PK, Kojima H, Martinez-Botas J, Sunehag AL, Chan L. Metabolic adaptations in the absence of perilipin: increased betaoxidation and decreased hepatic glucose production associated with peripheral insulin resistance but normal glucose tolerance in perilipin-null mice. J Biol Chem. 2004;279:35150-35158.

35. Sawada T, Miyoshi H, Shimada K, Suzuki A, Okamatsu-Ogura Y, Perfield JW 2nd, Kondo T, Nagai S, Shimizu C, Yoshioka N, Greenberg AS, Kimura K, Koike T. Perilipin overexpression in white adipose tissue induces a brown fat-like phenotype. PLoS One. 2010;5:e14006.

36. Miyoshi H, Souza SC, Endo M, Sawada T, Perfield JW 2nd, Shimizu C, Stancheva Z, Nagai S, Strissel KJ, Yoshioka N, Obin MS, Koike T, Greenberg AS. Perilipin overexpression in mice protects against diet-induced obesity. J Lipid Res. 2010;51:975-982.

37. Wang Y, Lam KS, Kraegen EW, Sweeney G, Zhang J, Tso AW, Chow WS, Wat NM, Xu JY, Hoo RL, Xu A. Lipocalin-2 is an inflammatory marker closely associated with obesity, insulin resistance, and hyperglycemia in humans. Clin Chem. 2007;53:34-41.

38. Kim KE, Jung Y, Min S, Nam M, Heo RW, Jeon BT, Song DH, Yi CO, Jeong EA, Kim H, Kim J, Jeong SY, Kwak W, Ryu do H, Horvath TL, Roh GS, Hwang GS. Caloric restriction of $\mathrm{db} / \mathrm{db}$ mice reverts hepatic steatosis and body weight with divergent hepatic metabolism. Sci Rep. 2016;6:30111.

39. Borkham-Kamphorst E, Drews F, Weiskirchen R. Induction of lipocalin-2 expression in acute and chronic experimental liver injury moderated by pro-inflammatory cytokines interleukin- $1 \beta$ through nuclear factor- $\kappa$ B activation. Liver Int. 2011;31:656-665.

40. Chawla A, Nguyen KD, Goh YP. Macrophage-mediated inflammation in metabolic disease. Nat Rev Immunol. 2011;11:738-749.

41. Jang E, Lee S, Kim JH, Kim JH, Seo JW, Lee WH, Mori K, Nakao K, Suk K. Secreted protein lipocalin-2 promotes microglial M1 polarization. FASEB J. 2013;27:1176-1190.

42. Yan QW, Yang Q, Mody N, Graham TE, Hsu CH, Xu Z, Houstis NE, Kahn BB, Rosen ED. The adipokine lipocalin 2 is regulated by 
obesity and promotes insulin resistance. Diabetes. 2007;56:25332540.

43. Law IK, Xu A, Lam KS, Berger T, Mak TW, Vanhoutte PM, Liu JT, Sweeney G, Zhou M, Yang B, Wang Y. Lipocalin-2 deficiency attenuates insulin resistance associated with aging and obesity. Diabetes. 2010;59:872-882.

44. Aldouri MA, Wonke B, Hoffbrand AV, Flynn DM, Ward SE, Agnew JE, Hilson AJ. High incidence of cardiomyopathy in beta-thalassaemia patients receiving regular transfusion and iron chelation: reversal by intensified chelation. Acta Haematol. 1990;84:113-117.

45. Hansen JB, Tonnesen MF, Madsen AN, Hagedorn PH, Friberg J, Grunnet LG, Heller RS, Nielsen AØ, Størling J, Baeyens L, Anker-
Kitai L, Qvortrup K, Bouwens L, Efrat S, Aalund M, Andrews NC, Billestrup N, Karlsen AE, Holst B, Pociot F, et al. Divalent metal transporter 1 regulates iron-mediated ROS and pancreatic $\beta$ cell fate in response to cytokines. Cell Metab. 2012;16:449-461.

46. Bankoglu EE, Seyfried F, Rotzinger L, Nordbeck A, Corteville C, Jurowich C, Germer CT, Otto C, Stopper H. Impact of weight loss induced by gastric bypass or caloric restriction on oxidative stress and genomic damage in obese Zucker rats. Free Radic Biol Med. 2016;94:208-217.

47. Arosio P, Levi S. Cytosolic and mitochondrial ferritins in the regulation of cellular iron homeostasis and oxidative damage. Biochim Biophys Acta. 2010;1800:783-792. 